\title{
SINDROME DISFÁGICA
}

\author{
Dysphagic syndrome
}

Ronaldo Mafia CUENCA, Daniélle Toniolo MALAFAIA, Gleim Dias SOUZA, Luciana Rodrigues Queiroz de SOUZA, Vicente Paulo da MOTTA, Mariliz Regina Antunes LIMA, Cacilda Joyce Ferreira da Silva GARCIA

ABCDDV/540

Cuenca RM, Malafaia DT, Souza GD, Souza LRQ, Motta VP, Lima MRA, Garcia CJFS. Sindrome disfágica. ABCD Arq Bras Cir Dig 2007; 20(2): 116-8.

RESUMO - Introdução - A disfagia é o principal sintoma das doenças do esôfago e é representada pela dificuldade em deglutir o alimento ingerido no trajeto da orofaringe até o estômago. Ela é queixa comum na prática clínica diária envolvendo várias especialidades na sua investigação. Métodos - Foi realizada revisão atualizada da literatura internacional através do Pubmed (www.pubmed.com) e nacional (www.lilacs.br) utilizando-se as seguintes palavras-chave: disfagia e transtornos da deglutição. A extensão do tema foi limitado aos seguintes enfoques: etiologia, diagnóstico e exames complementares mais aplicáveis à exploração propedêutica. Conclusão - Embora a literatura apresente bom entendimento do processo disfágico, no diagnóstico preciso de sua causa há freqüentemente necessidade de múltiplos exames complementares que associados fazem melhor entender o que acomete o paciente e orientará mais adequadamente a conduta a ser tomada

DESCRITORES - Transtornos da deglutição. Esofagopatias.

\section{INTRODUÇÃO}

Disfagia é o principal sintoma das doenças do esôfago sendo a sua caracterização muito importante para o manejo destes pacientes ${ }^{5}$. Ela é representada pela dificuldade em deglutir o alimento ingerido no trajeto da orofaringe até o estômago, podendo estar associada a outros sintomas como: regurgitação, aspiração traqueobrônquica, dor retroesternal independente do esforço físico (relacionada ou não à alimentação), pirose, rouquidão, soluço e odinofagia. Esta entidade é queixa comum na prática clínica diária envolvendo várias especialidades na sua investigação ${ }^{12,13}$.

\section{MÉTODO}

Foi realizada revisão atualizada da literatura internacional através do Pubmed (www.pubmed.com) e nacional (www.lilacs.br) utilizando-se a seguinte palavra-chave: transtornos da deglutição, obtendo-se grande quantidade de artigos dos quais 18 foram aproveitados nesta revisão por conterem abordagem ampla e detalhada do tema.

Trabalho realizado na Faculdade de Medicina do Planalto Central FAMEPLAC, Brasília, DF, Brasil.

Endereço para correspondência: Ronaldo Mafia Cuenca, e-mail: rmcuenca@uol.com.br

\section{REVISÃO DA LITERATURA}

\section{Etiologia}

As causas de disfagia dividem-se em dois grandes grupos: 1) as originadas de anormalidades do funcionamento da musculatura do esôfago, que é chamada de disfagia neuromuscular, e 2) as de causa mecânica com comprometimento da luz do órgão por lesões da sua própria parede ou por compressão extrínseca.

Outra causa de disfagia, mas que não está relacionada às doenças do esôfago é a disfagia orofaríngea, que é sintoma inespecífico causado por grupo de enfermidades estruturais da orofaringe. O paciente manifesta dificuldade no trânsito dos alimentos desde a boca até o esôfago, ou seja, no início da fase involuntária da deglutição ${ }^{16}$. Esta entidade clínica tem grande importância quando associada às doenças cerebrovasculares devido ao risco de pneumonia aspirativa ${ }^{6}$. Pacientes submetidos à laringectomia que apresentam disfagia no pós-operatório, têm como principal causa pseudodivertículos criados em conseqüência da técnica cirúrgica empregada ${ }^{14}$.

$\mathrm{Na}$ disfagia neuromuscular podem ser encontradas alterações anatômicas ou funcionais das próprias fibras musculares lisas ou esqueléticas, do sistema nervoso central ou periférico, e do sistema nervoso entérico, responsável pelos movimentos de todo o sistema digestório. Estas alterações neuromusculares podem ocorrer por doenças primárias do esôfago ou secundárias a distúrbios sistêmicos. 
As alterações neuromusculares podem estar presentes em qualquer segmento anatômico do corpo do esôfago e seus esfíncteres, podendo envolver um ou mais segmentos ao mesmo tempo ${ }^{4}$. Podem aparecer em qualquer idade, não sendo muito freqüentes em jovens e, quando da presença nessa faixa, sua causa na maioria das vezes é inespecífica ${ }^{3}$.

A disfagia mecânica surge quando a elasticidade ou o diâmetro do esôfago é reduzido a menos de $50 \%$ da sua luz, sendo queixa freqüente para alimentos sólidos. Exemplo dela é o câncer do esôfago ${ }^{10}$.

Quando a disfagia ocorre no pós-operatório da doença do refluxo gastroesofágico, a principal causa é neuromuscular quer resultante da manipulação técnica do esôfago para expô-lo durante a confecção da fundoplicatura, quer pré-existente no pré-operatório e não diagnosticada quando, então, ocorre piora com a manipulação cirúrgica. Alguns autores recomendam o estudo manométrico dos pacientes que serão submetidos ao tratamento cirúrgico para escolha da melhor opção técnica para evitar o agravamento de disfagia eventualmente pré-existente $e^{8,9,11}$.

A presença da disfagia em diferentes faixas etárias sugere etiologias diferentes. Nas pessoas com menos de 50 anos, as causas mais freqüentes são em ordem de probabilidade: esofagite de refluxo, acalásia, tumores benignos, carcinomas, compressão extrínseca e esclerodermia. Nas pessoas com mais de 50 anos, são: carcinoma (principal causa), esofagite de refluxo, anel esofágico, acalásia e espasmo esofágico difuso (Quadro 1).

\begin{tabular}{|c|c|}
\hline \multicolumn{2}{|l|}{ Mecânicas } \\
\hline \multicolumn{2}{|l|}{ Intrínsecas } \\
\hline - & Estenose péptica benigna \\
\hline - & Carcinoma \\
\hline • & Anel esofágico \\
\hline & Tumores benignos \\
\hline & Lesão cáustica \\
\hline & Divertículo de Zenker \\
\hline \multicolumn{2}{|l|}{ Extrínsecas } \\
\hline - & Compressões extrínsecas tumorais \\
\hline • & Bócios mergulhantes \\
\hline • & Alterações anatômicas vasculares \\
\hline \multicolumn{2}{|c|}{ Neuromusculares } \\
\hline \multicolumn{2}{|c|}{ Transtornos do músculo liso } \\
\hline - & Acalásia \\
\hline - & Espasmo esofágico difuso \\
\hline • & Esclerodermia \\
\hline \multicolumn{2}{|c|}{ Transtornos do músculo estriado/liso } \\
\hline - & Acalásia cricofaríngea \\
\hline - & Polimiosite \\
\hline \multicolumn{2}{|c|}{ Transtornos neurológicos } \\
\hline • & Doenças desmielinizantes \\
\hline
\end{tabular}

QUADRO 1 - Causas mais freqüentes da síndrome disfágica

\section{Diagnóstico}

Durante a realização da anamnese deve-se caracterizar cada um dos sintomas de maneira criteriosa para que se possa formular boa hipótese diagnóstica e decidir pelo melhor exame complementar. O tipo do alimento associado à disfagia deve ser bem explorado durante a história clínica, porque quando a disfagia é para líquidos, na fase inicial, a causa deve ter origem neuromuscular e quando para sólidos com progressão para líquidos as causas mais prováveis são de origem mecânica. Disfagias tanto para sólidos como para líquidos desde o início, sugerem doenças neuromusculares degenerativas como miastenia grave e síndrome de Parkinson por interferirem na função propulsora da junção neuromuscular e $20 \%$ de seus portadores apresentam-na como primeiro sintoma ${ }^{2}$.

Outra informação importante é o tempo de duração e a evolução da disfagia. A sintomatologia da síndrome disfágica de origem neuromuscular tem caráter crônico e intermitente; a evolução é longa e progressiva para alimentos sólidos e tem suas principais causas nas doenças benignas.

A localização da disfagia também é útil e pode ajudar na definição da sua causa. Quando as queixas estão localizadas ao nível do apêndice xifóide, pode-se estar frente à acalásia, ou doenças do esôfago distal. Nas queixas localizadas nos segmentos mais altos do tórax, além das doenças esofágicas, deve-se pensar em doenças dos órgãos extra-esofágicos vizinhos ao esôfago como traquéia, tireóide e tumores cervicais não-esofágicos.

Informações sobre manipulação cirúrgica prévia na transição esôfago-gástrica é de fundamental importância devido à alta incidência de disfagia no pós-operatório das fundoplicaturas ${ }^{17}$.

A dor retroesternal associada à disfagia para alimentos sólidos desde o início dos sintomas, é forte indício de doenças motoras como espasmo esofágico difuso.

A associação da disfagia com rouquidão pode indicar tumores esofágicos com invasão secundária da laringe ou do nervo laríngeo recorrente, e nos casos em que a rouquidão precede a disfagia pode ser tumores primários da laringe.

Quando no início da história clínica, os pacientes referem azia e queimação retroesternal antes da disfagia, então, estenose péptica e dismotilidades esofágicas por refluxo podem ser pensadas.

Durante a realização do exame físico devem-se procurar alterações músculoesqueléticas sistêmicas, neurológicas, pulmonares, tireoidianas e cutâneas.

\section{Exames complementares}

A endoscopia digestiva alta está indicada em todos os casos de síndrome disfágica, tanto para o diagnóstico como para a realização da biópsia de lesões existentes. Nas causas mecânicas, ela quase sempre faz diagnóstico e também pode ajudar no tratamento curativo ou paliativo.

Os exames radiológicos que podem ajudar no diagnóstico da síndrome disfágica são: seriografia esofagogastroduodenal, videofluoroscopia da deglutição e videodeglutograma. Eles definem a forma do esôfago e a alteração da motilidade existente e o comprometimento da luz do esôfago. Dos exames radiológicos, a videofluoroscopia da deglutição é importante para elucidação de doenças motoras 
e deve ser o primeiro exame a ser solicitado quando da suspeita de disfagia de origem neuromuscular, sendo sensível e específico para quantificar a severidade da disfagia ${ }^{7}$. $\mathrm{Na}$ suspeita da disfagia orofaríngea a videofluoroscopia da deglutição ou deglutograma é o primeiro exame a ser solicitado ${ }^{6,16}$. Também o é na investigação da disfagia na pediatria, que nestes casos é considerado padrão-ouro da investigação ${ }^{15}$.

O estudo manométrico do esôfago permite o registro das pressões na luz do órgão. As medidas de pressões são obtidas através de sondas colocadas em diferentes pontos, para que se possam obter informações do corpo esofágico e dos esfíncteres. Ele é muito importante para diagnóstico dos casos de disfagia neuromuscular.

A pHmetria esofágica de 24 horas é exame de eleição para os casos onde a endoscopia não registra anormalidade da mucosa e o paciente tem manifestações extra-esofágicas de doenças do refluxo gastroesofágico, como tosse, rouquidão, dor de garganta e bronco-aspirações noturnas. Através dela pode-se verificar a presença de refluxo, que é representado por aumento de acidez em nível cervical. Ele é realizado com a colocação de sonda especial para dosagem do $\mathrm{pH}$ que possui aberturas em determinados pontos, permitindo medir a acidez desta luz gástrica, até o esôfago cervical, passando por pontos intermediários no corpo do esôfago. Os achados são armazenados em um aparelho especial que fica fixado na cintura do paciente, com ele permanecendo nas 24 horas realizando todas suas atividades habituais do dia.

A utilização da ecoendoscopia ou ultra-sonografia endoscópica pode ser útil nos casos de lesões com comprometimento da parede esofágica sem lesão da mucosa do órgão. Através dela, é possível definir se a lesão é extrínseca ao esôfago ou se tem origem nas próprias camadas da parede do esôfago.

\section{CONCLUSÃO}

Embora a literatura apresente bom entendimento do processo disfágico, no diagnóstico preciso de sua causa há freqüentemente necessidade de múltiplos exames complementares que associados fazem melhor entender o que acomete o paciente e orientará mais adequadamente a conduta a ser tomada.

Cuenca RM, Malafaia DT, Souza GD, Souza LRQ, Motta VP, Lima MRA, Garcia CJFS. Dysphagic syndrome. ABCD Arq Bras Cir Dig 2007; 20(2): $116-8$.

ABSTRACT - Background - Dysphagia is the main symptom of diseases of the esophagus, being manifested by deglutition difficulties, which reaches the oropharynx and stomach pathway. Is a common daily complaint in the clinical practice, involving numerous specialties regarding its investigation. Methods - An international literature review was made using Pubmed (www.pubmed.com) and a national utilizing (www.lilacs.br) the following keywords: dysphagia and deglutition disorders. The extension of the subject was limited to the following areas: etiology, diagnosis and complementary exams which were aplicable to exploratory propedeutics. Conclusion - Eventhough the literature presents a good understanding of the dysphagic process, the need for multiple complementary exams is frequent during diagnosis, developing a better understanding of what the patient has, adequately orienting which conduct should be taken.

HEADINGS - Deglutition disorders. Esophageal diseases.

\section{REFERÊNCIAS}

1. Bethge N, Vakil N. A prospective trial of a new self-expanding plastic stent for malignant esophageal obstruction. Am J Gastroenterol. 2001;96:1350-4.

2. Cabrera CS, Álvarez MR, Pérez MA, Calle JM, García B. Myasthenia gravis: the otolaryngologist's perspective. Am J Otolaryngol. 2002;23:169-72.

3. Dantas RO. Alterações motoras do esôfago no paciente jovem com disfagia. GED Gastroenterol Endosc Dig. 2000;3:128-32.

4. Domingues GR, Lemme EM. Manifestações clínicas dos distúrbios motores esofagianos. Rev Bras Med. 2000;4:239-45.

5. Domingues GR, Lemme EM. Diagnóstico diferencial dos distúrbios motores esofagianos pelas características da disfagia. Arq Gastroenterol. 2001;1:148.

6. Gomes GF, Campos ACL, Pisani JC, Macedo Filho ED, Ribas Filho JM, Malafaia O, Czeczko NG. Sonda nasoenteral, aspiração traqueal e pneumonia aspirativa em pacientes hospitalizados com doença cérebro-vascular complicada por disfagia orofaríngea. ABCD Arq Bras Cir Dig. 2003;16:189-92.

7. Han TR, Paik NJ, Park JW. Quantifying swallowing function after stroke: a functional dysphagia scale based on videofluoroscopic studies. Arch Phys Med Rehabil. 2001;82:677-82.

8. Heider TR, Behms KE, Koruda MJ, Shaheen NJ, Lucktong TA, Bradshaw B, Farrell TM. Fundoplication impoves disordered esophageal motility. J Gastrointest Surg. 2003; 7:159-63.

9. Heider TR, Farrel TM, Kircher AP, Colliver CC, Koruda MJ, Behms KE. Complete fundoplication is not associated with increased dysphagia in patients with abnormal esophageal motility. J Gastrointest Surg. 2001;5:36-41.

10. Homs MY, Eijkenboom WM, Coen VL, Haringsma J, Blankenstein M, Kuipers EJ, Siersema PD. High dose rate brachytherapy for the palliation of malignant dysphagia. Radiother Oncol. 2003;66:327-32.

11. Liaño AD, Oteiza F, Ciga MA, Aizcorbe M, Trujillo R, Cobo F. Nonobstructive dysphagia and recovery of motor disorder after antireflux surgery. Am J Surg. 2003;185:103-7.
12. Lôbo BL, Cury RC, Alves P, Batista LD, Hachul MT, Mendonça RA, Lima SS. Videodeglutograma: indicações, técnica de exame, achados radiográficos normais e anormais. An Paul Med Cir. 2001;1:4-10.

13. McKeown MJ, Torpey DC, Gehm WC. Non-invasive monitoring of functionally distinct muscle activations during swallowing. Clin Neurophysiol. 2002;113:354-66.

14. Oursin C, Pitzer G, Fournier P, Bongartz G, Steinbrich W. Anterior neopharyngeal pseudodiverticulum: a possible cause of dysphagia laryngectomized patients. Clin Imaging. 1999;23:15-8.

15. Pinto RB, Almeida ST, Delgado SE, Cruz L. Avaliação multidiciplinar da criança com disfagia. Rev Bras Nutr Clín. 2001;4:139-43.

16. Quera PR, Defilippi CC. Disfagia orofaríngea. Acta Gastroenterol Latinoam. 2001;1:26-35.

17. Tatum RP, Shi G, Manka MA, Brasseur JG, Joehl RJ, Kahrilas PJ. Bolus transit assessed by an esophageal stress test in postfundoplication dysphagia. J Surg Res. 2000;91:56-60.

18. Vakil N, Morris AI, Marcon N, Segalin A, Peracchia A, Bethge N, Zucarro G, Bosco JJ, Jones WF. A prospective, randomized, controlled trial of covered expandable metal stents in the palliation of malignant esophageal obstruction at the gastroesophageal junction. Am J Gastroenterol. 2001;96:1791-6.

Conflito de interesse: não há Fonte financiadora: não há Recebido para publicação em: 03/01/2007 Aceito para publicação em: 05/03/2007 